\title{
Detection of sea-serpent field lines in sunspot penumbrae ${ }^{\star}$
}

\author{
A. Sainz Dalda ${ }^{1,2}$ and L. R. Bellot Rubio ${ }^{3}$ \\ 1 THEMIS S.L., C/Vía Láctea s/n, 38200 La Laguna, Tenerife, Spain \\ e-mail: asainz@themis.iac.es \\ 2 Instituto de Astrofísica de Canarias, C/Vía Láctea s/n, 38200 La Laguna, Tenerife, Spain \\ 3 Instituto de Astrofísica de Andalucía, CSIC, Apdo. 3004, 18080 Granada, Spain \\ Received 21 November 2007 / Accepted 19 December 2007
}

\section{ABSTRACT}

\begin{abstract}
Aims. We investigate the spatial distribution of magnetic polarities in the penumbra of a spot observed very close to disk center. Methods. High angular and temporal resolution magnetograms taken with the Narrowband Filter Imager aboard Hinode are used in this study. They provide continuous and stable measurements in the photospheric Fe I 630.25 line for long periods of time. Results. Our observations show small-scale, elongated, bipolar magnetic structures that appear in the mid penumbra and move radially outward. They occur in between the more vertical fields of the penumbra, and can be associated with the horizontal fields that harbor the Evershed flow. Many of them cross the outer penumbral boundary, becoming moving magnetic features in the sunspot moat. We determine the properties of these structures, including their sizes, proper motions, footpoint separation, and lifetimes.

Conclusions. The bipolar patches can be interpreted as being produced by sea-serpent field lines that originate in the mid penumbra and eventually leave the spot in the form moving magnetic features. The existence of such field lines has been inferred from Stokes inversions of spectropolarimetric measurements at lower angular resolution, but this is the first time they are imaged directly. Our observations add another piece of evidence in favor of the uncombed structure of penumbral magnetic fields.
\end{abstract}

Key words. sunspots - Sun: magnetic fields - Sun: photosphere - magnetohydrodynamics (MHD) - plasmas

\section{Introduction}

Significant progress in the understanding of the penumbra has been made through high resolution magnetograms and Dopplergrams (Title et al. 1993; Schlichenmaier \& Schmidt 2000; Langhans et al. 2005; Rimmele \& Marino 2006), spectropolarimetric measurements (Degenhardt \& Wiehr 1991; Sánchez Almeida \& Lites 1992; Lites et al. 1993; Stanchfield et al. 1997; Rüedi et al. 1999; Westendorp Plaza et al. 2001; Schlichenmaier \& Collados 2002; Mathew et al. 2003; Bellot Rubio et al. 2004; Borrero et al. 2005, 2006; Sánchez Cuberes et al. 2005; Beck 2006; Jurčák et al. 2007), and forward modeling (Martínez Pillet 2000; Müller et al. 2002). Today it is agreed that the penumbra consists of magnetic fields having different inclinations and strengths, as proposed by Solanki \& Montavon (1993) in their uncombed penumbral model. One particularly relevant result derived from spectropolarimetry is that some field lines return to the solar interior well within the penumbra. The modest angular resolution of groundbased polarimeters has precluded an unambiguous direct detection of such opposite-polarity field lines or the study of their temporal evolution.

Also, in recent years there has been an increased interest in determining the relation between moving magnetic features (MMFs; Harvey \& Harvey 1973) and penumbral magnetic fields. There is growing observational evidence that bipolar MMFs are the continuation of the more horizontal magnetic fields of the penumbra in the sunspot moat (Sainz Dalda \& Martínez Pillet 2005; Ravindra 2006;

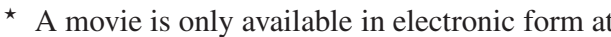
http: //www . aanda.org
}

Cabrera Solana et al. 2006; Kubo et al. 2007a), but a clear picture has not yet emerged due to insufficient angular resolution.

Establishing the small-scale organization of the magnetic field in the penumbra is crucial to shed light on these issues, and also to distinguish between competing models of the penumbra. Here we investigate the distribution of magnetic polarities in and around sunspots using magnetograph observations taken with Hinode (Kosugi et al. 2007). The unprecedented resolution and stability of these measurements allow us to follow the evolution of the penumbral fine structure in polarized light for hours.

\section{Observations}

Our study is based on longitudinal magnetograms acquired with the Narrowband Filter Imager (NFI; Tarbell et al. 2008) aboard Hinode. On November 14, 2006, the NFI observed the isolated spot AR 10923 from 07:10 to 09:40 UT and from 11:00 to 17:10 UT. The instrument was tuned to measure the Stokes $I$ and $V$ signals of Fe I $630.25 \mathrm{~nm}$ at $-120 \mathrm{~m} \AA$ from line center. The magnetograms cover a field of view (FOV) of $328^{\prime \prime} \times 164^{\prime \prime}$, and have an irregular cadence of 1-5 min. The effective pixel size of the measurements is 0 .' $^{\prime} 16$, which gives a spatial resolution of about $0{ }^{\prime} 32$. The spectral resolution of the NFI is $90 \mathrm{~m} \AA$. The spot crossed the central meridian around 10 UT, reaching a minimum heliocentric angle of $6^{\circ}$.

The data have not been corrected for instrumental effects because of difficulties in constructing accurate flatfields. We therefore restrict our analysis to the polarity of the magnetic field. The polarity should be insensitive to spatial variations in the instrument transmission because it is obtained as the difference of two intensity measurements. 
Single wavelength magnetograms, such as the ones used here, may fail to retrieve the polarity of the field in the presence of large Doppler shifts or multilobed Stokes $V$ spectra. These profiles are common in sunspot penumbrae because of their strong Evershed flows (e.g., Schlichenmaier \& Collados 2002; Bellot Rubio et al. 2007). However, in sunspots close to disk center the horizontal Evershed flow does not significantly shift the Stokes $V$ spectra. Only near the outer edge of the spot, where field lines are thought to return to the surface, may the flow produce redshifts in both the center-side and the limb-side penumbra. Our measurements were taken in the blue wing of Fe I $630.25 \mathrm{~nm}$, so Doppler shifts to the red cannot change the sign of the magnetogram signal. In addition, the small heliocentric angle of the spot means that projection effects are negligible. For these reasons, negative polarities in the magnetograms truly correspond to field lines pointing to the solar surface, whereas positive polarities indicate fields directed away from the sun.

The magnetograms have been aligned by cross-correlation to create a movie for the 07:10-17:10 UT period with a gap between 09:40 and 11:00 UT (AR10923.mpg, available as Supplementary Material). This presentation of the data makes it easier to follow the temporal evolution of the polarization signals in the penumbra. The movie covers a FOV of $112^{\prime \prime} \times 112^{\prime \prime}$, and has a regular cadence of $5 \mathrm{~min}$.

\section{Results}

The upper panel of Fig. 1 shows a magnetogram of AR 10923. The polarity of the spot is negative, but for clarity purposes we have reversed the sign of the magnetograms to treat it as a positive-polarity spot. With a color scale saturating at $\pm 500 \mathrm{DNs}$, the filamentary structure of the penumbra is clearly seen. Also conspicuous is the existence of many negative (black) polarity patches well within the penumbra. They are observed not only on the limb side, but also on the center side of the spot. The positions of some of these structures are indicated with circles.

A careful inspection of the magnetograms reveals that most of the negative-polarity patches are associated with positivepolarity structures located next to them but farther from the umbra. When detected, the positive-polarity patch has a stronger magnetogram signal than the background (which is also positive). Usually, the two patches appear elongated and narrow. The magnetogram movie shows that these opposite-polarity pairs move radially outward as a single entity.

The bottom panels of Fig. 1 give examples of bipolar penumbral structures. We have included contours of the umbra and the penumbra (obtained from the intensity images) to help identify the radial distances at which they are seen. Figure 1 illustrates the following properties of these structures:

- they appear in the mid penumbra (e.g., \#3, 5, and 10);

- they occur between penumbral filaments with enhanced positive signals (see the arrows in the panels of Fig. 1);

- they exhibit a filamentary structure (e.g, \#3, 6, and 7);

- the lengths of the individual patches are usually in the range $2-3^{\prime \prime}$, although some extreme cases of $5^{\prime \prime}$ have been detected. Their mean width is $1^{\prime \prime} .5$, but they can be as narrow as 0.5 .

To investigate the temporal evolution of the bipolar structures in more detail we use time-slice diagrams. They represent the polarization signals observed along the trajectories of the patches as a function of time. The width of the paths is 0 '. $^{\prime} 16$, corresponding to one pixel in the magnetograms. Figure 2 displays time-slice diagrams for filaments $\# 1,3,9$, and 10. As can be seen, the typical separation between the two opposite-polarity patches is on the order of $1-2^{\prime \prime}$. They move with velocities in the range $0.3-0.4 \mathrm{~km} \mathrm{~s}^{-1}$, although others propagate as fast as $0.7 \mathrm{~km} \mathrm{~s}^{-1}$. No significant velocity variations are detected. Occasionally, more than one bipolar pair seem to travel along the same filament, one after the other. This appears to be the case in the four examples of Fig. 2, but we cannot draw a definite conclusion due to the gap between 9:40 and 11:00 UT.

The time-slice diagrams show that the positive and negative patches often cross the outer penumbral boundary (represented by the dotted lines). In the case of filaments \#1 and \#9, the two patches travel a distance of some 3-6" in the moat. The crossing of the penumbral edge does not modify the speed of these features, but they adopt a more roundish shape outside the spot.

The magnetogram movie demonstrates that, at any given time, up to 15-20 bipolar structures may coexist in the penumbra. They are observed all around the umbra, with no preferred direction. Their lifetime can be as short as 30 min or longer than $7 \mathrm{~h}$. Usually, the former are very weak and do not reach the outer penumbral boundary.

\section{Discussion and conclusions}

The appearance and coherent motion of the bipolar structures described above gives a strong impression that they represent the two footpoints of magnetic field lines having the shape of a sea serpent. The opposite-polarity patches are observed in regions of weak positive magnetogram signals, flanked by penumbral filaments with stronger signals. Since the spot was virtually at disk center, weaker signals indicate more horizontal and/or weaker fields. These fields are precisely the ones that carry the Evershed flow. Thus, the bipolar structures occur in the more inclined fields of the penumbra, surrounded by the vertical fields of the spines (Lites et al. 1993).

We suggest that the opposite-polarity patches are the manifestation of $\mho$-loop perturbations of the field lines driving the Evershed flow. The negative-polarity patch, the one closer to the umbra, contains field lines returning to the solar surface, while the leading positive-polarity patch represents the re-emergence of the same field lines. The fact that the magnetogram signal is stronger in the positive-polarity patch compared with its surroundings indicates more vertical fields than both the spines and intra-spines, consistent with the idea that it represents the upstream footpoint of a $\mho$-loop. There have been reports of negative-polarity field lines from MDI magnetograms (Sainz Dalda \& Martínez Pillet 2005; Ravindra 2006), SST magnetograms (Langhans et al. 2005), and Hinode measurements (Ichimoto et al. 2007; Bellot Rubio et al. 2007), but this is the first time they are (a) observed in the center-side penumbra; (b) associated with positive-polarity counterparts that move together toward the edge of the spot; and (c) identified as the footpoints of sea-serpent field lines. Very likely, these structures are the ones producing the Evershed clouds discovered by Shine et al. (1994) and analyzed in detail by Cabrera Solana et al. (2007, 2008). The scenario favored by the present observations is similar to that resulting from the moving tube simulations of Schlichenmaier (2002). In the simulations, penumbral field lines associated with strong Evershed flows develop sea-serpent shapes during their rise from the spot magnetopause.

Our measurements show with unprecedented clarity that most of the opposite-polarity patches reach the edge of the spot and enter the moat. The transition is smooth, with no changes in the propagation speed. In other words, these structures are 

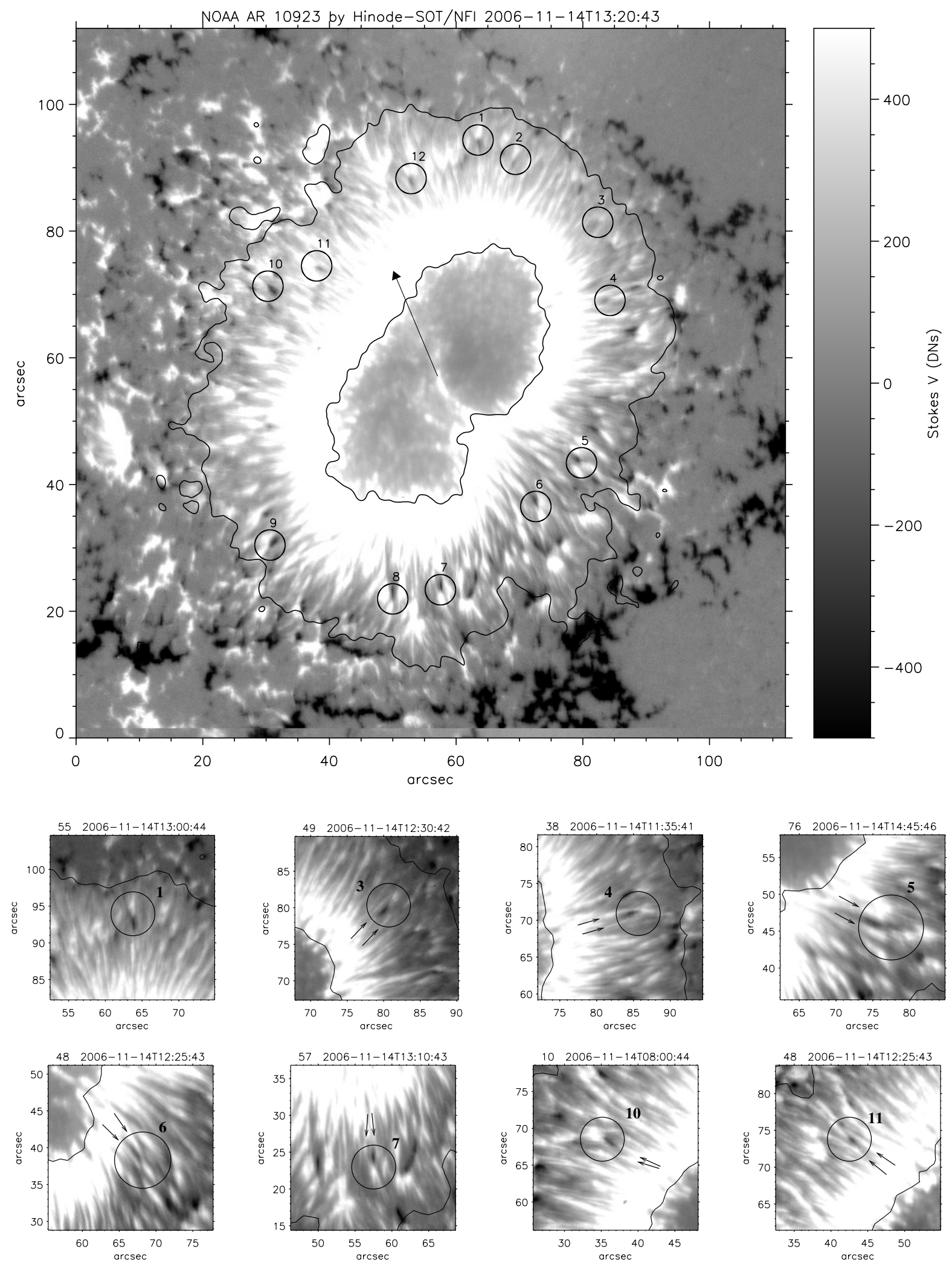

Fig. 1. Top: magnetogram (Stokes $V$ map) of AR 10923 with umbral and penumbral contours overlaid. The magnetogram signal has been reversed to treat the spot as if it were of positive polarity. The direction to disk center is marked with an arrow. The circles indicate a number of negativepolarity signals in the penumbra. The magnetogram is saturated at \pm 500 data numbers (hereafter DNs). Bottom: $222^{\prime \prime} 5 \times 22^{\prime \prime} 5$ subfields showing some of the negative-polarity patches. Usually, they are associated with a positive-polarity structure farther from the umbra. The bipolar pairs occur in between filaments of positive polarity (indicated with arrows). The frame number and time of each magnetogram is given in the title. 

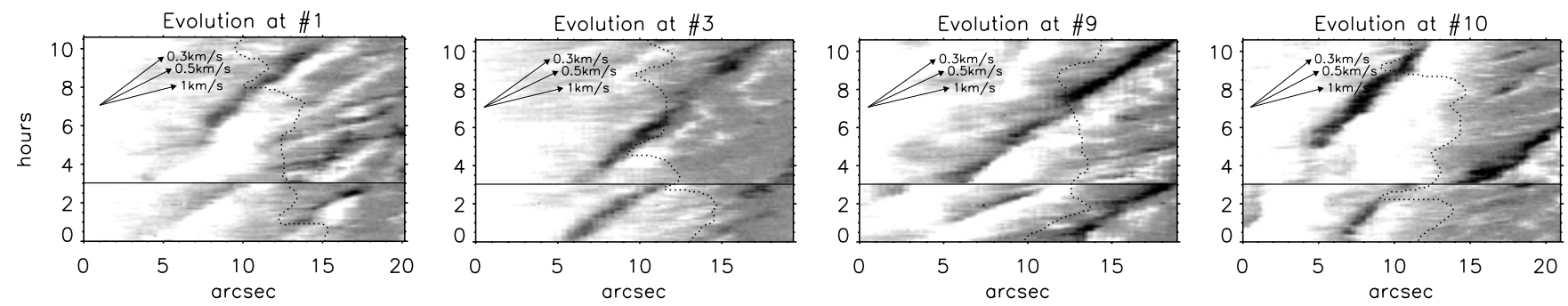

Fig. 2. Time-slice diagrams for filaments \#1,3,9, and 10. They have been created following the trajectories of the opposite-polarity patches defining the filaments. The horizontal solid lines mark the position of the gap between 9:40 and 11:00 UT. The dotted lines represent the instantaneous outer penumbral boundary. The arrows show the slopes of structures moving at $0.3,0.5$, and $1 \mathrm{~km} \mathrm{~s}^{-1}$.

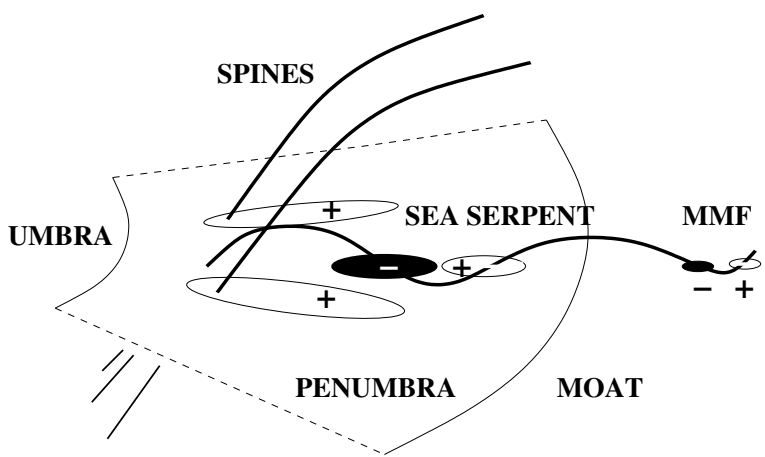

Fig. 3. Sketch of the penumbra showing sea-serpent field lines in between more vertical spine fields. The ovals indicate the polarity of the different structures when observed at disk center.

the precursors of the bipolar moving magnetic features observed around sunspots, confirming the results of Zhang et al. (2003), Sainz Dalda \& Martínez Pillet (2005), Cabrera Solana et al. (2006), and Kubo et al. (2007a,b). MMFs are the continuation of the penumbral fields that harbor the Evershed flow, as suggested theoretically by Schlichenmaier (2002), Thomas et al. (2002), and others. Their magnetic configuration must be that of a $\mho$-loop, since this is the shape of the field lines in the bipolar structures while they still reside in the penumbra. Zhang et al. (2007) and Cabrera Solana (2007) have deduced a similar configuration for bipolar MMFs.

Our interpretation of the observations is summarized in Fig. 3. We draw the inclined fields of the penumbra as sea serpents flanked by more vertical field lines representing the penumbral spines. The sea serpents propagate across the penumbra and reach the moat, where they become bipolar MMFs.

This magnetic configuration is in good agreement with the moving tube model of Schlichenmaier et al. (1998), which is essentially a siphon flow (Meyer \& Schmidt 1968; Degenhardt $1989,1991)$ with supercritical velocities. It remains to be seen whether subcritical and/or critical siphon flows (Thomas \& Montesinos 1993; Montesinos \& Thomas 1997), as well as other models of the penumbra, such as the turbulent pumping model (Thomas et al. 2002), the gappy penumbral model (Spruit \& Scharmer 2006; Scharmer \& Spruit 2006), or 3D simulations of sunspot penumbrae (e.g., Heinemann et al. 2007), can also explain the observations. In any event, it is clear that the detailed topology of these field lines must be inferred from spectropolarimetric data, necessarily at the same or better resolution than that provided by Hinode.

Acknowledgements. We thank all the scientists involved in the operation of Hinode as Chief Observers for their continuous support. Hinode is a Japanese mission developed and launched by ISAS/JAXA, with NAOJ as domestic partner and NASA and STFC (UK) as international partners. It is operated by these agencies in cooperation with ESA and NSC (Norway). This work has been partially funded by the Spanish MEC through projects ESP2006-13030-C06-01, ESP2006-13030-C06-02, AYA2004-05792, and AYA2007-66502.

\section{References}

Beck, C. 2006, Ph.D. Thesis, Alberts-Ludwig Universität, Freiburg, Germany Bellot Rubio, L. R., Balthasar, H., \& Collados, M. 2004, A\&A, 427, 319 Bellot Rubio, L. R., Tsuneta, S., Ichimoto, K., et al. 2007, ApJ, 668, L91 Borrero, J. M., Lagg, A., Solanki, S. K., \& Collados, M. 2005, A\&A, 436, 333 Borrero, J. M., Solanki, S. K., Lagg, A., Socas-Navarro, H., \& Lites, B. 2006, A\&A, 450, 383

Cabrera Solana, D. 2007, Ph.D. Thesis, University of Granada, Granada, Spain Cabrera Solana, D., Bellot Rubio, L. R., Beck, C., \& del Toro Iniesta, J. C. 2006, ApJ, 649, L41

Cabrera Solana, D., Bellot Rubio, L. R., Beck, C., \& del Toro Iniesta, J. C. 2007, A\&A, 475, 1067

Cabrera Solana, D., Bellot Rubio, L. R., Borrero, J. M., \& del Toro Iniesta, J. C. 2008, A\&A, 477, 273

Degenhardt, D. 1989, A\&A, 222, 297

Degenhardt, D. 1991, A\&A, 248, 637

Degenhardt, D., \& Wiehr, E. 1991, A\&A, 252, 821

Jurčák, J., Bellot Rubio, L., Ichimoto, K., et al. 2007, PASJ, 59, 601

Harvey, K., \& Harvey, J. 1973, Sol. Phys., 28, 61

Heinemann, T., Nordlund, A., Scharmer, G. B., \& Spruit, H. C. 2007, ApJ, 669, 1390

Ichimoto, K., et al. 2007, PASJ, 59, 593

Kosugi, T., Matsuzaki, K., Sakao, T., et al. 2007, Sol. Phys., 243, 3

Kubo, M., Shimizu, T., \& Tsuneta, S. 2007a, ApJ, 659, 812

Kubo, M., Ichimoto, K., Shimizu, T., et al. 2007b, PASJ, 59, 607

Langhans, K., Scharmer, G. B., Kiselman, D., Löfdahl, M. G., \& Berger, T. E. 2005, A\&A, 436, 1087

Lites, B. W., Elmore, D. F., Seagraves, P., \& Skumanich, A. P. 1993, ApJ, 418, 928

Martínez Pillet, V. 2000, A\&A, 361, 734

Mathew, S. K., Lagg, A., Solanki, S. K., et al. 2003, A\&A, 410, 695

Meyer, F., \& Schmidt, H. U. 1968, Z. Angew. Math. Mech., 48, 218

Montesinos, B., \& Thomas, J. H. 1997, Nature, 390, 485

Müller, D. A. N., Schlichenmaier, R., Steiner, O., \& Stix, M. 2002, A\&A, 393, 305

Ravindra, B. 2006, Sol. Phys., 237, 297

Rüedi, I., Solanki, S. K., \& Keller, C. U. 1999, A\&A, 348, L37

Rimmele, T., \& Marino, J. 2006, ApJ, 646, 593

Sainz Dalda, A., \& Martínez Pillet, V. 2005, ApJ, 632, 1176

Sánchez Almeida, J., \& Lites, B. W. 1992, ApJ, 398, 359

Sánchez Cuberes, M., Puschmann, K. G., \& Wiehr, E. 2005, A\&A, 440, 345

Scharmer, G. B., \& Spruit, H. C. 2006, A\&A, 460, 605

Schlichenmaier, R. 2002, Astron. Nachr., 323, 303

Schlichenmaier, R., \& Schmidt, W. 2000, A\&A, 358, 1122

Schlichenmaier, R., \& Collados, M. 2002, A\&A, 381, 668

Schlichenmaier, R., Jahn, K., \& Schmidt, H. U. 1998, A\&A, 337, 897

Shine, R. A., Title, A. M., Tarbell, T. D., et al. 1994, ApJ, 430, 413

Solanki, S. K., \& Montavon, C. A. P. 1993, A\&A, 275, 283

Spruit, H. C., \& Scharmer, G. B. 2006, A\&A, 447, 343

Stanchfield, D. C. H., Thomas, J. H., \& Lites, B. W. 1997, ApJ, 477, 485

Title, A. M., Frank, Zoe A., Shine, Richard A., et al. 1993, ApJ, 403, 780

Tarbell, T. D., et al. 2008, Sol. Phys., in preparation

Thomas, J. H., \& Montesinos, B. 1993, ApJ, 407, 398

Thomas, J. H., Weiss, N. O., Tobias, S. M., \& Brummell, N. H. 2002, Nature, 420,390

Westendorp Plaza, C., del Toro Iniesta, J. C., Ruiz Cobo, B., \& Martínez Pillet, V. 2001, ApJ, 547, 1148

Zhang, J., Solanki, S. K., \& Wang, J. 2003, A\&A, 399, 755

Zhang, J., Solanki, S. K., Woch, J., \& Wang, J. 2007, A\&A, 471, 1035 